\section{Comment on Recognition and confusion of the lowercase alphabet}

\section{DOUGLAS G. MILLOY \\ University of Calgary, Calgary, Alberta, Canada}

In a recent issue of this journal, Geyer (1977) presents an analysis of a full alphabet confusion matrix for lowercase letters in foveal vision. Geyer indicates that previous confusion data for the lowercase alphabet (Bouma, 1971) are inconclusive. Bouma presents two experiments in which the procedures used to control average correct performance differ substantially. In one experiment, letters are presented foveally, correct performance being limited to $50 \%$ by manipulating viewing distance. The second experiment manipulated the degree of eccentric exposure along the horizontal meridian, to achieve the desired level of performance. A forced-choice procedure was used in the latter experiment but not in the former, and the letter y was omitted from the stimulus set in the nonfoveal condition. Bouma concluded on subjective grounds that the foveal and nonfoveal matrices were sufficiently similar, and thus averaged the results across the foveal and nonfoveal confusion matrices in subsequent analyses. Geyer (1977) suggests that the comparability of processing between foveal and nonfoveal regions, based on identification performance, must be considered an empirical question.

Geyer attempts to answer this question by comparing the confusion matrix reported by Bouma (1971) for eccentric vision with the foveal matrix presented in Geyer (1977). He makes this comparison on the basis of the sum square deviation of (1) the foveal and nonfoveal matrices (2.08), and (2) the foveal matrix and a dummy matrix (1.79), "uniformly composed of 0.5 along main-diagonal cells and 0.02 elsewhere" (Geyer, 1977, p. 489). The use of this technique in the evaluation of confusion matrix data is not unprecedented. Townsend (1971a, 1971b) suggests the sum of squared deviations as an index of the accuracy of fit between predicted and empirical confusion matrices. However, caution must be exercised in the interpretation of this index. Geyer assumes this difference to be a significant one. The statistical basis for this assumption is not entirely clear. How large a difference is significant? Without a knowledge of the underlying distribution, such an inferential statement cannot be made.

Geyer concludes that the difference between sum square deviations may be regarded as evidence in support of the notion "that foveal and nonfoveal recognition processes differ qualitatively" (p. 489).
This statement is somewhat premature and may overstep the facts. No mention is made regarding the manner in which the missing data from Bouma's nonfoveal matrix are treated. If the row for $y$ ( $y$ was not presented) in Bouma's 25 by 26 matrix is replaced by inserting .00 in all of the cells, then the sum square deviation is artificially inflated. Some mention of the way in which this problem was dealt with is essential to the interpretation of the comparison.

Over and above the statistical question, there is perhaps a more fundamental criticism to be considered. The stimuli in Geyer's study were from the "Tactype Futura demi 5424" alphabet, a font without serifs. Geyer states that this type style was chosen "in order to minimize the influence of any particular type style idiosyncracy"' (p. 488). Bouma (1971) used letters of the typeface "Courier 10" (IBM), a font which has "pronounced serifs" (p. 460). An inspection of the two type styles reveals that there are considerable differences in structure between corresponding letter pairs.

A few examples will serve to illustrate the potential confound inherent in the comparison of different type styles. The letter "A" in "Tactype Futura demi 5424 " (a) shares very few features in common with the same letter in "Courier 10" (a) type. The similarity of Geyer's ( $a)$ to the letter $(0)$ is reflected in the $14 \%$ confusion response between these two when ( $a$ ) is the stimulus. Contrast with this the lack of confusion reported by Bouma, between the same two letters $(a)$ and $(0)$ when $(a)$ is the stimulus $(1 \%)$. A more compelling example is seen in the $31 \%$ confusion reported by Bouma when $(g)$ is the stimulus and $(q)$ the response. The same confusion occurs only $7 \%$ of the time for Geyer's ( $\mathbf{g}$ ) and ( $\mathbf{q}$ ). The difference in structure in and of itself, between these confusion pairs, might be an important contributing factor to the differences between the foveal and nonfoveal confusion matrices.

It seems questionable whether the differences reported by Geyer (1977) can be unequivocally attributed to differences in information processing between different retinal loci. It may be unreasonable to assume that differences in type style can be disregarded in this way. Maddox, Burnette, and Gutmann (1977) present data for uppercase dot matrix letters, which indicate that letter style may be an important variable altering the pattern of confusions. Further, research presented by Fisher, Monty, and Glucksberg (1969) suggests that comparison across confusion data derived from different procedures may not be valid. The comparison made by Geyer (1977) rests on the assumption that a basic "pattern of confusion" exists between the lowercase letters of the 
alphabet. This would imply that no differences in the pattern of confusions would exist between the two type styles presented foveally. On this basis, one could infer that any differences observed in the pattern of confusions between foveal and peripheral identification would be a function of processing differences across different retinal positions. However, the two type styles have yet to be shown perceptually equivalent. Neither has it been demonstrated that the pattern of confusion responses, even within type styles, is immutable, in the sense that we may regard the confusions as a function of underlying perceptual phenomena (Fisher et al., 1969).

If we were to accept the reasoning of Geyer regarding the significance of the sum square deviations in his study, we should also conclude that there are qualitative differences in processing between individuals and between an individual and group data, based on Townsend (1971a, 1971b). However, Townsend (1971b) suggests that both individual and group data were consistent in sensory confusions, differing only in terms of response biases-a very different interpretation indeed. It is suggested that were the type style differences eliminated, the difficulty of interpreting the sum square deviation index, independent of other considerations, would remain.

It seems evident from the preceding considerations that unequivocal support for the contention of differences in processing between foveal and nonfoveal regions has not been presented by Geyer (1977). Clearly, both type-style influences and retinal-position influences must be assessed directly rather than by assumption and across different experimental procedures.

\section{REFERENCES}

Bouma, H. Visual recognition of isolated lower-case letters. Vision Research, 1971, 11, 459-474.

Fisher, D. F., Monty, R. A., \& Glucksberg, S. Visual confusion matrices: Fact or artifact? Joumal of Psychology, 1969, 71, 111-125.

GeYer, L. H. Recognition and confusion of the lowercase alphabet. Perception \& Psychophysics, 1977, 22, 487-490.

Maddox, M. E., Burnette, J. T., \& Gutmann, J. C. Font comparisons for 5 x 7 dot matrix characters. Human Factors, 1977, 19. 89-93.

Tow NSEND, J. T. Theoretical analysis of an alphabetic confusion matrix. Perception \& Psychophysics, 1971, 9, 40-50. (a)

Townsend, J. T. Alphabetic confusion: A test of models for individuals. Perception \& Psychophysics, 1971, 9, 449-454. (b)

(Received for publication May 15, 1978; accepted June 15, 1978.) 